\title{
Bioinspired Assembly of Inorganic Nanoplatelets for Reinforced Polymer Nanocomposites
}

\author{
Tzung-Hua Lin, Wei-Han Huang, In-kook Jun and Peng Jiang \\ University of Florida \\ United States
}

\section{Introduction}

Steel and metal alloys have long been used for structural applications because they are both strong and flaw-tolerant. By contrast, ceramics, which are strong but not tolerant to surface flaws and cracks, and polymers, which are flaw-tolerant and can deform under applied stresses, are less favourable as structural materials. However, nature has resolved this dilemma by millions of years of biological evolution. For example, the nacreous layer of mollusk shells(Jackson, Vincent et al. 1988; Aksay, Trau et al. 1996; Smith, Schaffer et al. 1999), which is made up of relatively weak components (consisting of 95 volume \% of brittle aragonite platelets and 5 volume \% of soft biological macromolecules), shows unexpected toughness (resistance to cracking) and stiffness (resistant to deformation)(Barthelat 2007). The oriented assembly of aragonite platelets and the intricate brick-and-mortar nanostructure found in the nacreous layer have been attributed to the major reasons for the exceptional toughness and stiffness of nacres. This unusual combination of different mechanical properties inspires scientists to create strong and flaw-tolerant artificial materials that mimic the mechanical design principles found in nacres by combining platelet-like ceramic building blocks with polymeric matrices(Tang, Kotov et al. 2003; Podsiadlo, Kaushik et al. 2007; Bonderer, Studart et al. 2008).

Various bottom-up self-assembly techniques have been developed for creating biomimetic reinforced nanocomposites. Layer-by-layer (LBL) assembly of inorganic nanoplatelets and polyelectrolytes has recently been demonstrated as an efficient methodology in making reinforced polymer nanocomposites(Podsiadlo, Kaushik et al. 2007; Bonderer, Studart et al. 2008; Podsiadlo, Michel et al. 2008). The sequential adsorption of anionic montmorillonite clay platelets and poly(diallydimethylammonium) chloride polycation results in an organicinorganic hybrid material that has ultimate tensile strength ( 100 MPa) approaching to that of nacre ( 130 MPa)(Tang, Kotov et al. 2003). By treating with glutaraldehyde to improve the bonding and load transfer between clay platelets and polymer matrices, nanocomposites made by LBL assembly of montmorillonite clay platelets and poly(vinyl alcohol) show even higher tensile strength ( 400 MPa)(Podsiadlo, Kaushik et al. 2007). Ice-templated crystallization, gravitational sedimentation, centrifugation, as well as spin-coating have also been explored to assemble inorganic nanoplatelets into ordered structures(Almqvist, Thomson et al. 1999; Chen, Wang et al. 2008; Liu, Chen et al. 2008; Munch, Launey et al. 
2008). Unfortunately, these techniques are either time-consuming or require multiple steps to infiltrate inorganic assemblies with polymer. For example, LBL assembly is a relative slow process. Hundreds of bilayers need to be deposited to form composites with micrometer-scale thickness. In addition, the inevitable agglomeration of commonly used clay restrains nanoplatelets from forming highly aligned structures and therefore weakens the mechanical properties of the resulting nanocomposites.(Liu, Chen et al. 2008)

In this chapter, we will show that ordered assemblies of nanoplatelets can be achieved by utilizing electrophoretic deposition, which is the migration of charged particles under the influence of an applied electric field and has been widely used in assembling spherical colloids into highly ordered colloidal crystals(Braun and Wiltzius 1999; Holgado, GarciaSantamaria et al. 1999; Velev and Bhatt 2006). Organic-inorganic hybrid materials can be directly formed by electrophoretic co-deposition of inorganic platelets and organic matrix, or by sequential polymer infiltration in the interstitials of inorganic assemblies(Lin, Huang et al. 2009). Indeed, electrophoretic deposition has been extensively utilized for the deposition of ceramic particles because of its simplicity and controllability, such as bath composition and deposition parameters, and is an effective technique for rapid and scalable fabrication of nanocomposites over large areas(Zhitomirsky 2002). We will show that cathodic co-deposition of either non-ionic-type polymers or polyelectrolytes between nanoplatelets enable the deposition of organic-inorganic nanocomposites in a simple singlestep approach(Grandfield and Zhitomirsky 2008; Pang and Zhitomirsky 2008; Lin, Huang et al. 2009; Lin, Huang et al. 2009). This simple electrodeposition technique will significantly reduce the cost and increase the throughput of reinforced polymer nanocomposites.

\section{Experimental section}

\subsection{Materials and substrates}

All solvents and chemicals are of reagent grade and are used without further purification. Ultrapure water $\left(18.2 \mathrm{M} \Omega \mathrm{cm}^{-1}\right)$ is used directly from a Barnstead water system. Ethanol (200 proof) is purchased from Pharmaco Products. Hydrochloric acid (37\%), aluminum secbutoxide $(\geq 95 \%)$, and aluminum isopropoxide $(\geq 98 \%)$ are obtained from Aldrich. Ethoxylated trimethylolpropane triacrylate monomer (ETPTA, SR 454) is obtained from Sartomer. The photoinitiator, Darocur 1173 (2-hydroxy-2-methyl-1-phenyl-1-propanone), is provided by Ciba Specialty Chemicals. Two-part polydimethylsiloxane (PDMS, Sylgard 184) is provided by Dow Corning. Polyvinyl alcohol (PVA, Mw 89,000 98,000) and polyethylenimine (PEI, Mw $\sim 750,000$ ) are purchased form Aldrich. Indium tin oxide (ITO) coated glass substrates with sheet resistance of $8 \Omega$ are purchased from Delta Technologies. Gold electrodes are prepared by sputtering deposition of $20 \mathrm{~nm}$ of titanium and $200 \mathrm{~nm}$ of gold on glass slides.

\subsection{Instrumentation}

A kurt J. Lesker CMS-18 Multitarget Sputter is used for the sputtering deposition of titanium and gold. An EG\&G Model 273A potentiostat/galvanostat (Princeton Applied Research) is used for electrophoretic deposition. Transmission electron microscopy (TEM) is performed on a JEOL 2010F TEM. Scanning electron microscopy (SEM) is carried out on a JEOL 6335F FEG-SEM. A thin layer of gold is sputtered onto the samples prior to imaging. X-ray diffraction spectra are obtained with a Philips APD-3720 equipment. A Cu Ka ${ }_{1}(\lambda=1.540491$ $\AA$ ) radiation is scanned from $10^{\circ}$ to $70^{\circ}$ with a scan rate of $2.4^{\circ} / \mathrm{min}$. Atomic force 
microscopy (AFM) is carried out on a Digital Instruments Dimension 3100 unit. The zeta potential of gibbsite nanoplatelets is measured by a Brookhaven ZetaPlus Analyzer (Brookhaven Instrument Corporation). A standard spin coater (WS-400B-6NPP-Lite Spin Processor, Laurell) is used to spin-coat ETPTA monomer. The polymerization of monomer is carried out on a Pulsed UV Curing System (RC 742, Xenon). A HR4000 UV-Vis spectrometer (Ocean Optics) is used for optical transmission measurement. Tensile strength measurement are tested using an Instron model 1122 load frame upgraded with an MTS ReNew system and equipped with a $500 \mathrm{~g}$ load cell at a crosshead speed of $0.5 \mathrm{~mm} / \mathrm{min}$.

\subsection{Synthesis of inorganic gibbsite nanoplatelets}

The gibbsite nanoplatelets are synthesized by the following preparation method(Wierenga, Lenstra et al. 1998). To $1 \mathrm{~L}$ of ultrapure water, hydrochloric acid $(0.09 \mathrm{M})$, aluminum secbutoxide $(0.08 \mathrm{M})$, and aluminum isopropoxide $(0.08 \mathrm{M})$ are added. The mixture is stirred for 10 days and then heated in a polyethylene bottle in a water bath at $85^{\circ} \mathrm{C}$ for $72 \mathrm{~h}$. After cooling to room temperature, dispersions of gibbsite nanoplatelets are centrifuged at $3500 \mathrm{~g}$ for $6 \mathrm{~h}$ and the sediments are redispersed in deionized water. This process is repeated for five times to remove any unreacted reactants and also concentrate the as-synthesized nanoplatelets.

\subsection{Electrophoretic deposition}

Electrophoretic deposition of nanoplatelets is performed in a water-ethanol mixture in a sandwich cell placed horizontally. The bottom and the top of the cell are either an ITO or a gold electrode. PDMS is used as a spacer to get an active area of $1.5 \times 1.5 \mathrm{~cm}^{2}$ and a cell gap of $2.2 \mathrm{~mm}$.

\subsubsection{Electrophoretic deposition of gibbsite nanoplatelets}

The bath solution is gibbsite nanoplatelets dispersed in a water-ethanol mixture. 2 weight \% of aqueous suspensions of gibbsite nanoplatelets is used. 200-proof ethanol is added into the suspensions to make the volumetric ratio of ethanol to the aqueous suspension to be 2 . A constant voltage of $-2.5 \mathrm{~V}$ (ITO vs. Au) is applied for $20 \mathrm{~min}$ to deposit the positively charged gibbsite nanoplatelets onto the bottom ITO working cathode. A gold electrode is used as the top counter anode.

\subsubsection{Electrophoretic deposition of PVA-gibbsite nanocomposites}

Electrophoretic bath solution is prepared by mixing $1 \mathrm{~mL}$ of $5 \mathrm{wt}$ \% PVA aqueous solution, 9 $\mathrm{mL}$ of $2.0 \mathrm{wt}$ \% gibbsite nanoplatelet aqueous solution, and $20 \mathrm{~mL}$ of 200 -proof ethanol. Constant voltage of $-2.5 \mathrm{~V}$ (ITO vs. Au electrode) is applied to deposit gibbsite nanoplatelets and PVA on the bottom ITO working cathode. A gold electrode is used as the top counter anode.

\subsubsection{Electrophoretic deposition of PEI-gibbsite nanocomposites}

Electrophoretic bath solution is prepared by mixing $2 \mathrm{~mL}$ of $0.3 \mathrm{wt}$.\% PEI aqueous solution, $3 \mathrm{~mL}$ of $2.0 \mathrm{wt}$.\% gibbsite nanoplatelet aqueous solution, and $10 \mathrm{~mL}$ of 200-proof ethanol. Constant current of $0.3 \mathrm{~mA}$ is applied for $15 \mathrm{~min}$ to deposit gibbsite nanoplatelets and PEI on the bottom gold working cathode. An ITO electrode is used as the top counter anode. 


\section{Gibbsite nanoplatelets}

Gibbsite, aluminum hydroxide $\left(\mathrm{Al}(\mathrm{OH})_{3}\right)$, is an inorganic crystalline with stacking $\mathrm{Al}-\mathrm{OH}$ layers. Each $\mathrm{Al}^{3+}$ is surrounded by six hydroxyl groups and can be synthesized from aqueous aluminium alkoxide solutions by hydrothermal treatment. The as-synthesized gibbsite nanoplatelets have well-defined hexagon-shape with aspect ratio of around 10, which is close to that of natural aragonite platelets found in nacre(Jackson, Vincent et al. 1988). The reaction of surface hydroxyl groups with water makes the gibbsite nanoplatelets highly charged in water and alcoholic suspensions, providing strong electrostatic repulsion responsible for the observed colloidal stability. The structure of the gibbsite crystals suggests that the acidity of the A1-OH groups at the edges is different from that on the faces. The isoelectric points for edges and faces of platelets are $\mathrm{pH} \sim 7$ and $\mathrm{pH} \sim 10$, respectively, resulting in an inhomogeneous charge density and causing different types of flocculation: face-to-face, edge-to-face and edge-to-edge flocculation, depending on solution $\mathrm{pH}$. Gibbsite nanoplatelets have been widely used as a model system to exploit the liquid crystal phase transition in suspensions of plate-like particles(van der Kooij and Lekkerkerker 1998; van der Kooij, Kassapidou et al. 2000; van der Beek and Lekkerkerker 2004; Mourad, Wijnhoven et al. 2006). Opal-like columnar gibbsite colloidal crystals have been demonstrated by sedimentation(Brown, Clarke et al. 1998; van der Beek, Radstake et al. 2007).

A typical TEM image of purified gibbsite nanoplatelets is shown in Fig. 1. The diameter of the gibbsite nanoplatelets is estimated from TEM micrographs to be around $200 \mathrm{~nm}$. TEM images also reveal the nanoplatelets tend to align parallel to the surface of TEM grids. It is very rare to find nanoplatelets oriented perpendicularly to the TEM grid surface as shown by the red arrow pointing to such a particle in Fig. 1. Thickness of nanoplatelets ranges from 10 to $15 \mathrm{~nm}$, confirmed by AFM. As mentioned before, the purified gibbsite nanoplatelets are electrostatically stabilized and the zeta-potential $(\zeta)$ of the colloids in deionized water is measured to be $+40.5 \pm 2.3 \mathrm{mV}$ by fitting experimental data using Smoluchowski's model. This high surface charge makes the dispersions of gibbsite nanoplatelets stable and aggregated particles are rarely seen in TEM images. The selected area electron diffraction (SAED) patterns from a single platelet as shown in the inset of Fig. 1 indicate the as-made gibbsite nanoplatelets are single-crystal.

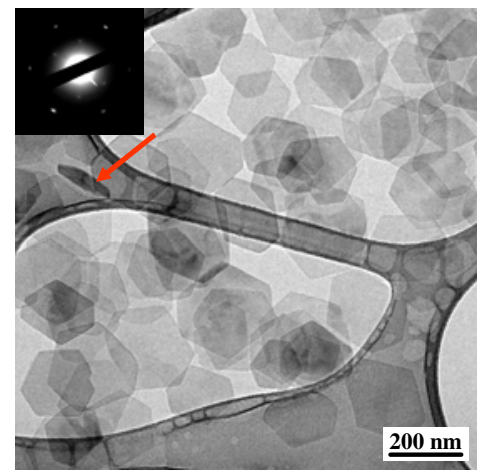

Fig. 1. TEM image of gibbsite nanoplatelets. The inset shows the electron diffraction patterns obtained from a single nanoplatelet. The red arrow points to a gibbsite nanoplatelet oriented perpendicularly to the TEM grid. Adapted from Lin, Huang et al. 2009. 


\section{ETPTA-gibbsite nanocomposites}

\subsection{Electrophoretic deposition of gibbsite nanoplatelets}

Electrophoretic deposition of positively charged gibbsite nanoplatelets is performed in a water-ethanol mixture and carried out using a horizontal parallel-plate sandwich cell, which consists of an ITO working electrode on the bottom, a gold counter electrode on the top, and a PDMS spacer ( $2.2 \mathrm{~mm}$ thick). Ethanol is used to reduce the dielectric constant of the solvent and therefore reduce the electric double-layer thickness of the nanoplatelets to promote colloidal coagulation on the gold electrode. Deionized water is added to the dispersions for bringing up the following cathodic reaction:

$$
2 \mathrm{H}_{2} \mathrm{O}+2 \mathrm{e}^{-} \rightarrow \mathrm{H}_{2}+2 \mathrm{OH}^{-} \quad \mathrm{E}^{0}=-0.828 \mathrm{~V}
$$

Since the surface of oxide particles could be positively or negatively charged, depending on solution $\mathrm{pH}$, the cathodic reaction above could result in a local $\mathrm{pH}$ increase at the electrode surface and thus lower the surface charge of positively charged gibbsite nanoplatelets to further assist colloidal coagulation. Contrary to the cathodic reaction for the electrodeposition of gibbsite nanoplatelets, the electrodeposition of negatively charged particles may require the following anodic reaction that results in a local $\mathrm{pH}$ decrease at the electrode:

$$
\mathrm{O}_{2}+4 \mathrm{H}^{+}+4 \mathrm{e}^{-} \rightarrow 2 \mathrm{H}_{2} \mathrm{O} \quad \mathrm{E}^{0}=-1.229 \mathrm{~V}
$$

Therefore, we disperse gibbsite nanoplatelets in a water-ethanol mixture with volumetric ratio of 1:2 to make the electrophoretic bath solution. The volume fraction of gibbsite particles is adjusted to $\sim 1 \%$. Without ethanol, no particle deposits are observed to adhere on the working electrode after disassembling the electrophoretic cell. The addition of ethanol also facilitates to reduce cracking and porosity in the electrodeposited films. The applied electric field strength is $\sim 1100 \mathrm{~V} / \mathrm{m}$. Besides parallel-plate geometry, electrodes can also be vertically inserted into the colloidal baths to conduct the electrophoretic deposition. As the gravitational sedimentation of gibbsite nanoplatelets is negligible during electrophoresis, uniform deposits are also resulted.

The deposits can be easily peeled off from the ITO surface by using a sharp razor blade, resulting in the formation of self-standing films as shown in Fig. 2A. The film is opaque and brittle. The side facing the ITO cathode is smoother than the side facing the suspension. The size of the resulting films is solely determined by the dimensions of the ITO electrode. Fig. $2 \mathrm{~A}$ depicts a sample with $1.6 \times 0.6$ in. $^{2}$ area deposited on a $2 \times 1$ inch $^{2}$ ITO electrode. Fig. $2 \mathrm{~B}$ shows a top-view SEM image of the suspension-side of the sample in Figure $2 \mathrm{~A}$. The hexagonal gibbsite nanoplatelets are densely packed and aligned parallel to the electrode surface. The alignment of gibbsite nanoplatelets is further confirmed by the layered structure as shown in the cross-sectional SEM image of Fig. 2C. Another convincing evidence of the orientated deposition comes from the X-ray diffraction (XRD) patterns shown in Fig. 2D. Only (002) and (004) peaks are observed in the XRD spectrum. As the crystallographic $c$-axis of single-crystal gibbsite is normal to the platelet surfaces, the (002) and (004) reflection are from gibbsite platelets oriented parallel to the electrode surface(Cullity 1978). Analysis of the half-height width of the (002) peak with the Scherrer equation yields an average platelet thickness of $15.1 \mathrm{~nm}$, agreeing with AFM measurement. 


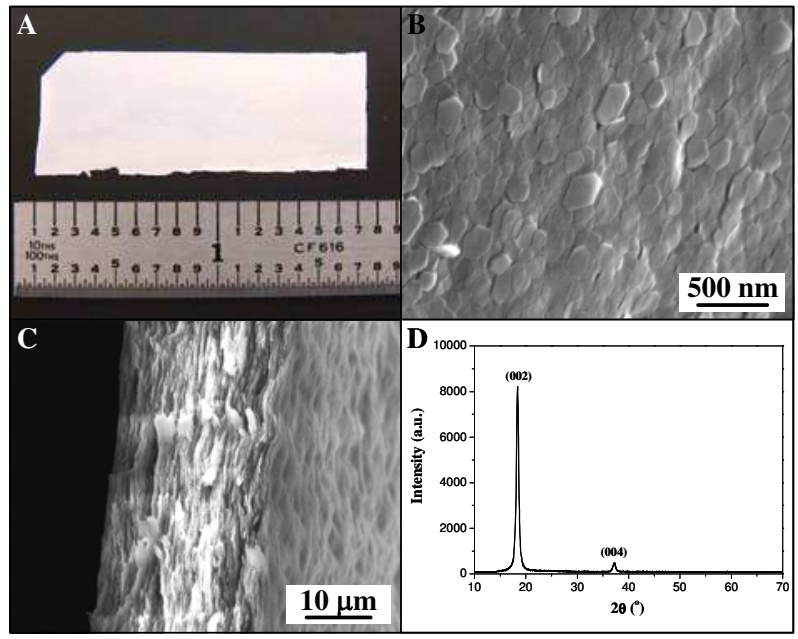

Fig. 2. Electrophoretic assembly of gibbsite nanoplatelets. (A) Photograph of a free-standing gibbsite film. (B) Top-view SEM image of the sample in (A). (C) Cross-sectional view of the same sample. (D) XRD patterns of the gibbsite film in (A). Adapted from Lin, Huang et al. 2009.

The oriented deposition of gibbsite nanoplatelets in a direct-current (dc) electric field can be understood by considering the charge distribution on the gibbsite surfaces due to different isoelectric points at faces $(\mathrm{pH} \sim 10)$ and edges $(\mathrm{pH} \sim 7)$. The $\mathrm{pH}$ of the bath in the electrophoretic experiments is close to 7 , resulting in positively charged surfaces and almost neutral edges. Therefore, the applied electric field exerts a force only on the surfaces of the gibbsite platelets and Brownian motion could provide sufficient torque to re-orient perpendicular particles to face the ITO electrode. Once being close to the electrode, the gibbsite nanoplatelets will be forced to align parallel to the electrode surface as this orientation is more energetically favorable than the perpendicular one. If the duration of the electrophoretic process is long enough, almost all gibbsite platelets can be deposited on the ITO electrode.

\subsection{Filling nanoplatelet assemblies with ETPTA}

After oriented deposition, polymer-gibbsite nanocomposites can then be made by filling the interstitials between the aligned nanoplatelets with photo-curable monomers, followed by photopolymerization. We choose a non-volatile monomer, ethoxylated trimethylolpropane triacrylate (ETPTA, M.W. 428, viscosity $60 \mathrm{cps}$ ), to form the nanocomposites. The monomer with 1\% photoinitiator (Darocur 1173, Ciba-Geigy) is spin-coated at $4000 \mathrm{rpm}$ for $1 \mathrm{~min}$ to infiltrate the electroplated gibbsite film and then polymerized by exposure to ultraviolet radiation. The resulting nanocomposite film becomes highly transparent (Fig. 3A) due to the matching of refractive index between the gibbsite platelets and the polymer matrix. The normal-incidence transmission measurement as shown in Fig. 3B shows the free-standing nanocomposite film exhibits high transmittance $(>80 \%)$ for most of the visible wavelengths. As the reflection $(R)$ from an interface between two materials with refractive index $\mathrm{n}_{1}$ and $\mathrm{n}_{2}$ is governed by Fresnel's equation(Macleod 2001): 


$$
\mathrm{R}=\left[\left(\mathrm{n}_{1}-n_{2}\right) /\left(n_{1}+n_{2}\right)\right]
$$

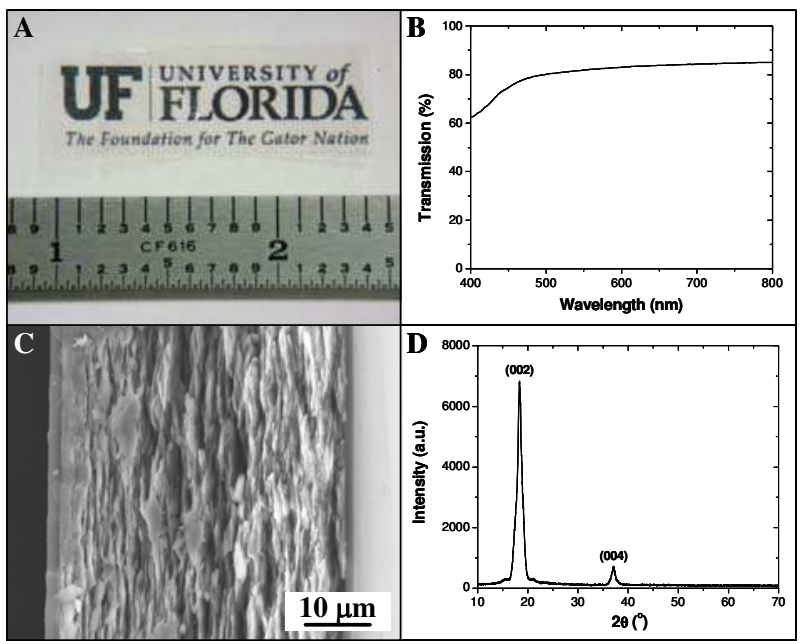

Fig. 3. Free-standing gibbsite-ETPTA nanocomposite. (A) Photograph of a transparent nanocomposite film. (B) Normal-incidence transmission spectrum of the sample in (A). (C) Cross-sectional SEM image of the same film. (D) XRD patterns of the same sample. Adapted from Lin, Huang et al. 2009.

we can estimate the normal-incidence reflection from each air-nanocomposite interface to be about $4 \%$. Thus, the optical scattering and absorption caused by the nanocomposite itself is ca. $10 \%$. This suggests the polymer matrix has infiltrated most interstitial spaces between the aligned gibbsite nanoplatelets. The cross-sectional SEM image in Fig. 3C shows the nanocomposite retains the layered structure of the original electroplated gibbsite film. Thin wetting layers of ETPTA ( $1 \mu \mathrm{m}$ thick) are observed on the surfaces of the film. The oriented arrangement of the nanoplatelets is also maintained throughout the polymer infiltration process as confirmed by the distinctive (002) and (004) peaks of the XRD spectrum shown in Fig. 3D.

\subsection{Composition analysis}

The ceramic weight fraction of the ETPTA-gibbsite nanocomposite film is determined by thermogravimetric analysis (TGA) as shown in Fig. 4. From the TGA curve and the corresponding weight loss rate, it is apparent that two thermal degradation processes occur. One happens at $\sim 250^{\circ} \mathrm{C}$ and corresponds to the degradation of the polymer matrix; while another occurs at $\sim 350^{\circ} \mathrm{C}$ and is due to the decomposition reaction of gibbsite:

$$
2 \mathrm{Al}(\mathrm{OH})_{3} \rightarrow \mathrm{Al}_{2} \mathrm{O}_{3}+3 \mathrm{H}_{2} \mathrm{O}
$$

Based on the residue mass percentage (45.65\%) and assuming the ash is solely $\mathrm{Al}_{2} \mathrm{O}_{3}$, we can estimate the weight fraction of gibbsite nanoplatelets in the original nanocomposite film to be $\sim 0.70$. Considering the density of gibbsite $\left(\sim 2.4 \mathrm{~g} / \mathrm{cm}^{3}\right)$ and ETPTA $\left(\sim 1.0 \mathrm{~g} / \mathrm{cm}^{3}\right)$, the volume fraction of gibbsite nanoplatelets in the nanocomposite is ca. 0.50 . The complete 
infiltration of ETPTA between the electroplated gibbsite platelets is further confirmed by the selective dissolution of gibbsite in a $2 \%$ hydrochloric acid aqueous solution. This results in the formation of a self-standing porous membrane with stacked hexagon-shaped pores, which are negative replica of the assembled gibbsite platelets.

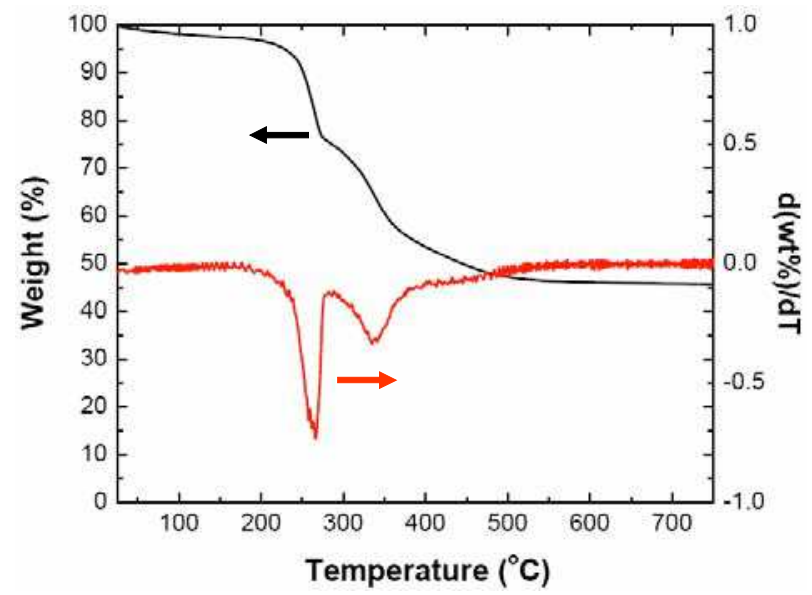

Fig. 4. Thermogravimetric analysis of a gibbsite-ETPTA nanocomposite. Adapted from Lin, Huang et al. 2009.

\subsection{Mechanical test}

The mechanical properties of the biomimetic polymer nanocomposites are evaluated by tensile tests. We compare the tensile strength for three types of thin films, including pure ETPTA, gibbsite-ETPTA, and TPM-modified gibbsite-ETPTA. The surface hydroxyl groups of gibbsite nanoplatelets can be easily modified by reacting with 3-(trimethoxysilyl)propyl methacrylate (TPM) through the well-established silane coupling reaction. This results in the formation of surface-modified particles with dangling acrylate bonds that can be crosslinked with the acrylate-based ETPTA matrix. The colloidal stability and the surface charge of the resulting nanoplatelets are not affected by this surface modification process as confirmed by TEM and zeta potential measurement. Fig. 5 shows the tensile stress versus strain curves for the above three types of films. The gibbsite-ETPTA nanocomposite displays $\sim 2$ times higher strength and $\sim 3$ times higher modulus when compared with pure ETPTA polymer. Even more remarkable improvement occurs when TPM-gibbsite platelets are crosslinked with the ETPTA matrix. We observe $\sim 4$ times higher strength and nearly one order of magnitude higher modulus than pure polymer. This agrees with early studies that reveal the crucial role played by the covalent linkage between the ceramic fillers and the organic matrix in determining the mechanical properties of the artificial nacreous composites.

We also conduct a simple calculation to evaluate if the measured mechanical properties of the gibbsite-ETPTA nanocomposites are reasonable. For a polymer matrix having a yield shear strength $\tau_{\mathrm{y}}$ and strong bonding to gibbsite nanoplatelet surface (e.g., TPM-modified gibbsites), the tensile strength of the composite $\left(\sigma_{c}\right)$ can be calculated using the volume fraction of nanoplatelets $\left(V_{\mathrm{p}}\right)$, the nanoplatelet aspect ratio $(\mathrm{s})$, and the tensile strength of the nanoplatelets $\left(\sigma_{\mathrm{p}}\right)$ and of the polymer matrix $\left(\sigma_{\mathrm{m}}\right)$, as(Bonderer, Studart et al. 2008) 


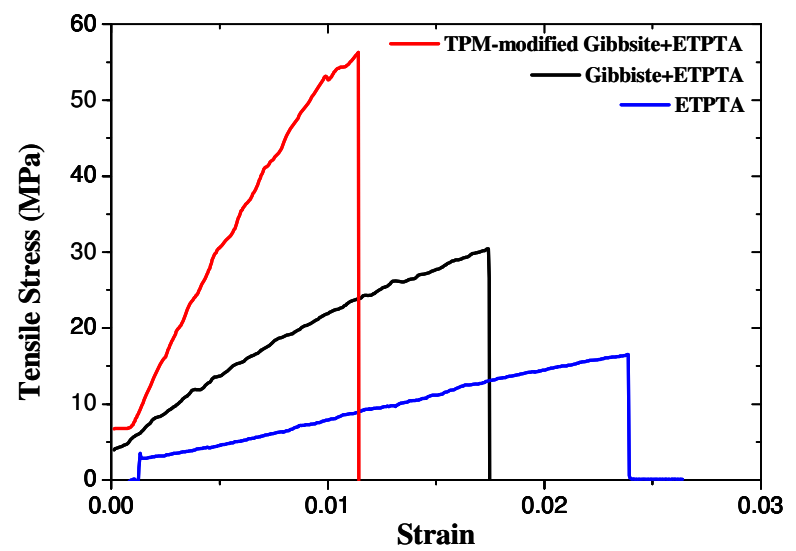

Fig. 5. Tensile stress versus strain curves for plain ETPTA film, ETPTA-gibbsite nanocomposite, and TPM-modified ETPTA-gibbsite nanocomposite. Adapted from Lin, Huang et al. 2009.

$$
\sigma_{\mathrm{c}}=\mathrm{aV}_{\mathrm{P}} \sigma_{\mathrm{P}}+\left(1-\mathrm{V}_{\mathrm{P}}\right) \sigma_{\mathrm{m}}
$$

For the gibbsite nanoplatelet which has a relatively small aspect ratio ( $\sim 12$ to 18 ), the factor $a$ in equation 3 can be estimated as

$$
\alpha=\tau_{y} s / 2 \sigma_{P}
$$

From the above TGA analysis, the volume fraction of gibbsite nanoplatelets in the polymer nanocomposite is $\sim 0.50$. If we take $s=15$, equation 3 can then be simplified as

$$
\sigma_{\mathrm{c}}=3.75 \tau_{\mathrm{y}}+0.5 \sigma_{\mathrm{m}}
$$

For acrylate-based polymer (like ETPTA), the yield shear strength should be close to its tensile strength. Equation 7 can further be simplified as $\sigma_{\mathrm{c}} \sim 4.25 \sigma_{\mathrm{m}}$. This indicates that the strength of the nanocomposite is about fourfold of the strength of the polymer matrix, agreeing with our experimental result.

\section{PVA-gibbsite nanocomposites}

\subsection{Single-step electrophoretic deposition of PVA-gibbsite nanocomposites}

The electrophoretic deposition of PVA-gibbsite nanocomposites is also carried out using the same parallel sandwich cell as described above. The high-molecular weight PVA (Mw $89,000-98,000)$ is neutrally charged in the electrophoretic bath and can be adsorbed on the surfaces of gibbsite nanoplatelets as water-soluble binders to cement electrodeposited gibbsite nanoplatelets together and also prevent the deposits from cracking. Fig. 6A shows a photograph of a PVA-gibbsite nanocomposite formed on an ITO cathode. The film can be easily peeled off from the electrode surface by using a sharp razor blade. The resulting selfstanding film is flexible and transparent, which is different from gibbsite deposits. Optical transmission measurement at normal-incidence shows the film exhibits $60-80 \%$ transmittance for most of the visible wavelengths. Top-view SEM image in Fig. 6B illustrates 
the gibbsite nanoplatelets are preferentially oriented with their crystallographic $c$-axis perpendicular to the electrode surface. It is very rare to find edge-on platelets. The ordered layered structure is clearly evident from the cross-sectional SEM images as shown in Fig. 6C and 6D.

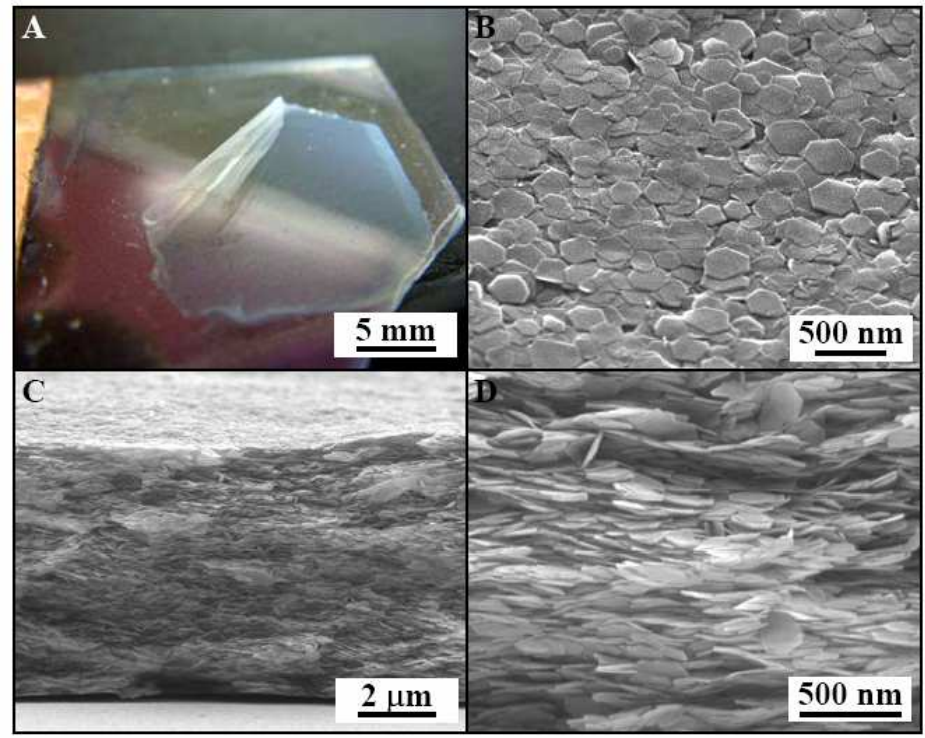

Fig. 6. Electrodeposited PVA-gibbsite nanocomposite. (A) Photograph of a composite film on an ITO electrode. (B) Top-view SEM image of the sample in (A). (C) Cross-sectional SEM image of the sample in (A). (D) Magnified cross-sectional image. Adapted from Lin, Huang et al. 2009.

\subsection{XRD and TGA analysis of PVA-gibbsite nanocomposites}

The oriented assembly of high-aspect-ratio gibbsite nanoplatelets is further confirmed by XRD. Fig. 7 displays a XRD spectrum of an electrodeposited PVA-gibbsite nanocomposite on an ITO electrode. The diffraction peaks from (222), (400), (441), and (662) planes of the ITO substrate are clearly appeared. Other than ITO diffraction peaks, we only observe (002) and (004) peaks from gibbsite single crystals. As the crystallographic c-axis of singlecrystalline gibbsite is normal to the platelet surfaces, the (002) and (004) reflection are from gibbsite platelets oriented parallel to the electrode surface. This strongly supports the macroscopic alignment of gibbsite nanoplatelets in the electrophoretically deposited nanocomposites.

Thermogravimetric analysis is used to determine the weight fraction of the inorganic phase in the electrodeposited nanocomposites. Fig. 8 shows the TGA curve and the corresponding weight loss rate for the PVA-gibbsite nanocomposite film. An apparent thermal degradation process occurs at $\sim 250^{\circ} \mathrm{C}$ that corresponds to the degradation of the PVA matrix and the decomposition reaction of gibbsite as shown in Equation 4 . Based on the residue mass percentage $(53.96 \%)$ and assuming the ash is solely $\mathrm{Al}_{2} \mathrm{O}_{3}$, we can estimate the weight fraction of gibbsite nanoplatelets in the original nanocomposite film to be 0.825 . 


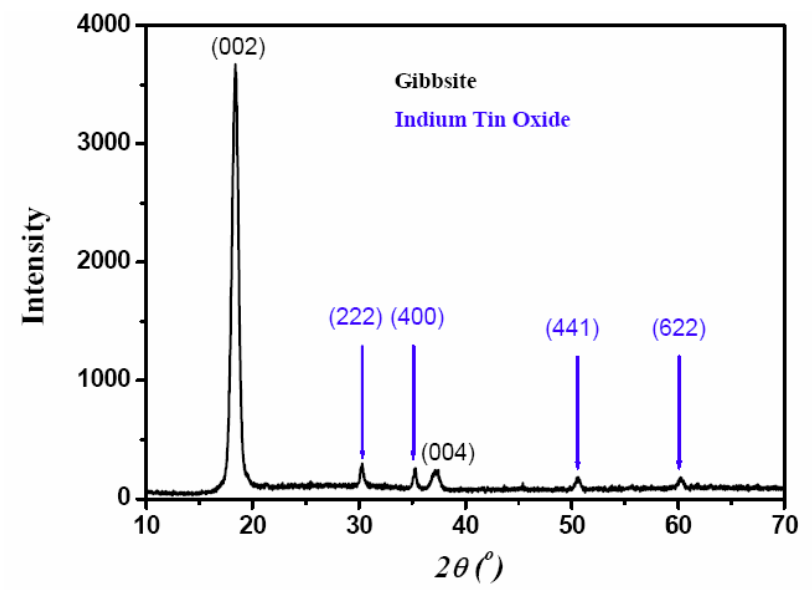

Fig. 7. XRD patterns of an electrodeposited PVA-gibbsite nanocomposite on an ITO electrode. Adapted from Lin, Huang et al. 2009.

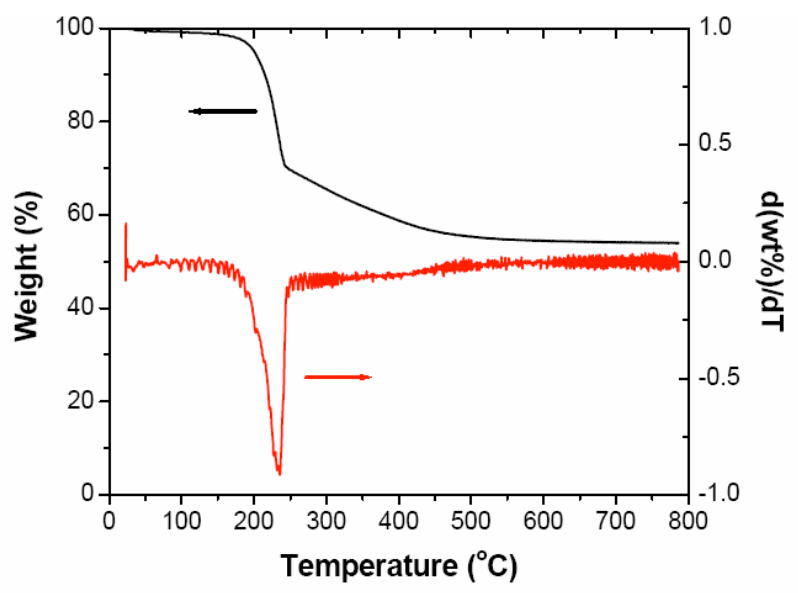

Fig. 8. Thermogravimetric analysis of PVA-gibbsite nanocomposites. Adapted from Lin, Huang et al. 2009.

\section{PEl-gibbsite nanocomposites}

Polyethyleneimine, which is a weak polyelectrolyte and contains amine groups, is positively charged under the electrophoretic conditions. The gibbsite nanoplatelets with a small amount of PEI are well dispersed in a water-ethanol mixture solution due to the electrostatic repulsion between particles. However, adding a larger amount of PEI leads to the agglomeration of gibbsite nanoplatelets. To allow the electrophoresis at a controlled deposition rate, as well as the formation of ordered layered structure, gibbsite nanoplatelets must be stabilized in suspensions. Therefore the influence of the PEI concentration on the stability of gibbsite is studied by measuring particle size distribution and zeta-potential. 


\subsection{Stability of PEl-gibbsite dispersions}

To prepare the testing solution, $(6-\mathrm{n}) \mathrm{mL}$ of $2.0 \mathrm{wt} \%$ gibbsite solution is mixed with $\mathrm{n} \mathrm{mL}$ of $0.3 \mathrm{wt} \%$ PEI aqueous solution, where $\mathrm{n}=0,1,2,3,4$, and 5 . The weight ratio (PEI to gibbsite, $\mathrm{R})$ is calculated as $(\mathrm{n} \times 0.3) /[(6-n) \times 2]$. Fig. 9 shows the size distribution of gibbsite nanoplatelets at different $\mathrm{R}$ values measured by laser diffraction. The average diameter of the as-synthesized gibbsite nanoplatelets $(R=0)$ is $150 \mathrm{~nm}$ (Fig. 9A), which is smaller than that observed from TEM images. The random mismatch of the surface of nanoplatelets to the incident laser beam reduces the effective diffraction area, resulting in a smaller average diameter. Fig. 9B shows that no significant change in the particle size distribution is observed when a small amount of PEI is added $(R=0.03)$. However, further increasing of PEI concentration, as shown in Fig. $9 \mathrm{C}$ and $9 \mathrm{D}(\mathrm{R}=0.075$ and 0.75 , respectively), leads to a larger particle diameter resulting from the flocculation of nanoplatelets. The flocculation at high polyelectrolyte concentration can be explained by the increase in ionic strength, which leads to the decrease in the electrical double-layer thickness and the instability of the colloids. Depletion flocculation also plays an important role. At a high polymer concentration, the polymer concentration gradient between the inter-particle gap and the remainder of the solution generates an osmotic pressure difference, forcing solvent flows out of the gap until particles flocculate(Dietrich and Neubrand 2001).
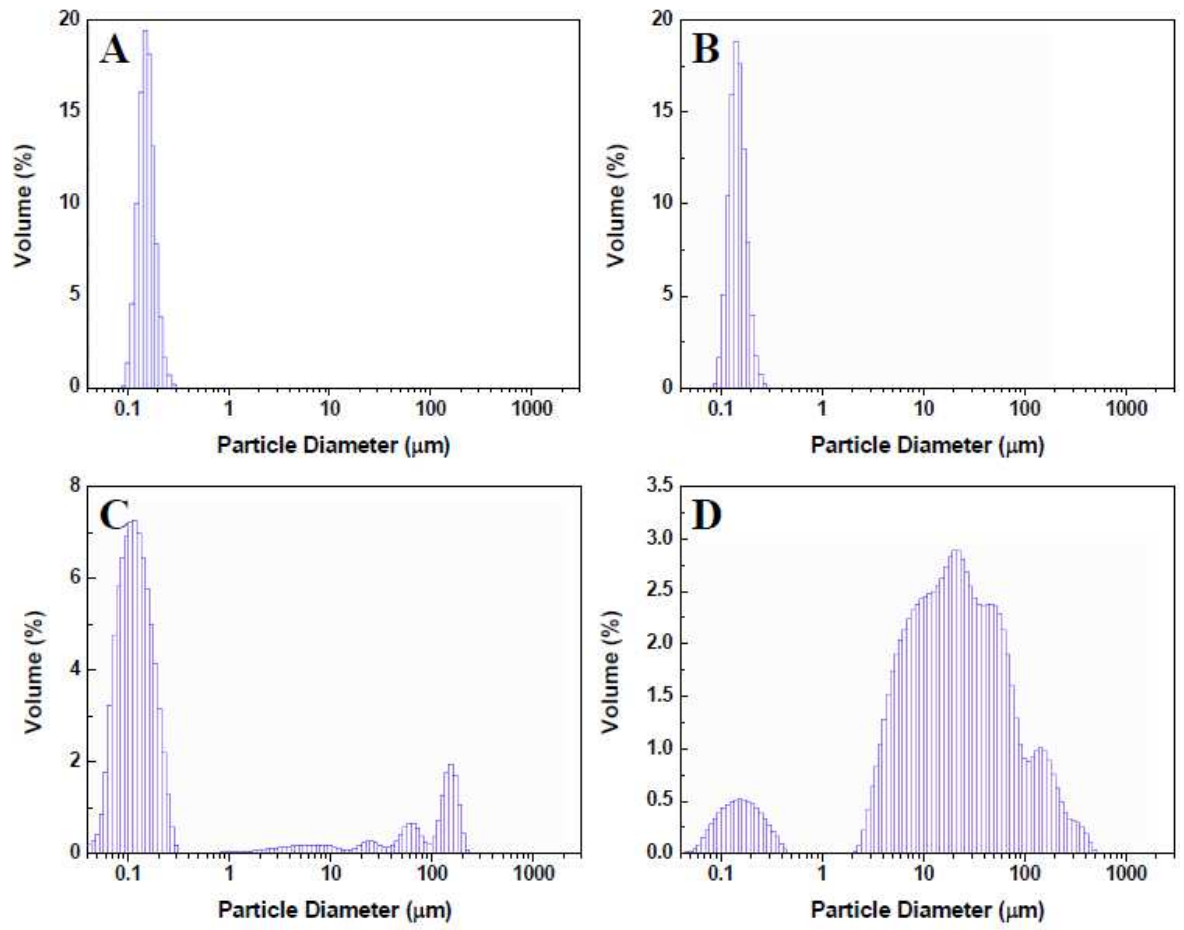

Fig. 9. Particle size distribution of nanoplatelet suspensions at different PEI/gibbsite weight ratios. (A) $R=0$, (B) $R=0.03,(C) R=0.075$, and (D) $R=0.75$. Adapted from Lin, Huang et al. 2009. 
Electrophoretic mobility and zeta-potential of nanoplatelets in PEI-gibbsite suspensions with different $R$ values are shown in Fig. 10. Zeta-potential is obtained by fitting experimental data using Smoluchowski's model. The increase of the electrophoretic mobility and zeta-potential when a small amount of PEI is added ( $\mathrm{R}$ from 0 to 0.03 ) is due to the contribution of highly charged PEI that possesses a zeta-potential of $\sim+60 \mathrm{mV}$ in water at neutral $\mathrm{pH}$. Further increasing of PEI concentration results in the decreasing of electrophoretic mobility and zetapotential due to the particle flocculation as shown in Fig. 9.

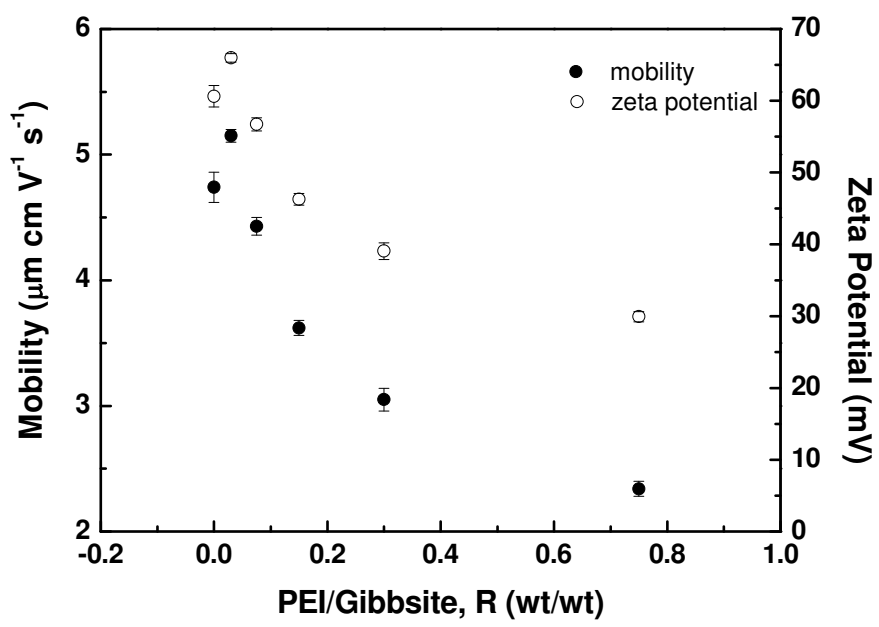

Fig. 10. Electrophoretic mobility and corresponding zeta-potential of nanoplatelets at different PEI/ gibbsite weight ratio. Adapted from Lin, Huang et al. 2009.

\subsection{Single-step electrophoretic deposition of PEI-gibbsite nanocomposites}

The electrophoretic deposition of PEI-gibbsite nanocomposite is again performed using a parallel-plate cell. The positively charged nanoplatelets are attracted toward the bottom $\mathrm{Au}$ cathode by the electrical force. As gibbsite nanoplatelets have positively charged surface and almost neutral edges under the electrophoretic conditions, the electric force tends to re-orient the gibbsite nanoplatelets to face the electrode. The positively charged PEI molecules are also electrophoretically migrated toward the cathode together with gibbsite and simultaneously sandwiched between nanoplatelets, forming PEI-gibbsite nanocomposite. Ethanol is added to promote particle coagulation by squeezing the electrical double-layer thickness of the gibbsite nanoplatelets. The high $\mathrm{pH}$ near the cathode also helps to coagulate nanoplatelets, as well as neutralize the protonated PEI macromolecules. Top-view SEM images in Fig. 11A and 11B show that the electrodeposited nanoplatelets are preferentially oriented with their crystallographic c-axis perpendicular to the electrode surface. The hexagonal shape and the size of the platelets can be clearly seen in Fig. 11B. Cross-sectional SEM images showed in Fig. $11 \mathrm{C}$ and 11D provide further evidence of the ordered layered structure.

\subsection{XRD and TGA analysis of PEl-gibbsite nanocomposites}

XRD spectrum of the PEI-gibbsite nanocomposite on an Au electrode is shown in Fig. 12. The diffraction peak from the (002) plane of gibbsite single crystals is clearly appeared. Comparing to previous results, which show diffraction peaks from both (002) and (004) 
planes of gibbsite crystals, the weaker diffraction peak from (004) plane is overlapped with the strong diffraction peak of Au. The (004) diffraction peak can be clearly seen by simply replacing $\mathrm{Au}$ electrode with $\mathrm{Pt}$ (not shown here). As the (002) and (004) diffraction are originated from gibbsite platelets oriented parallel to the electrode surface, the oriented assembly of nanoplatelets is further confirmed.

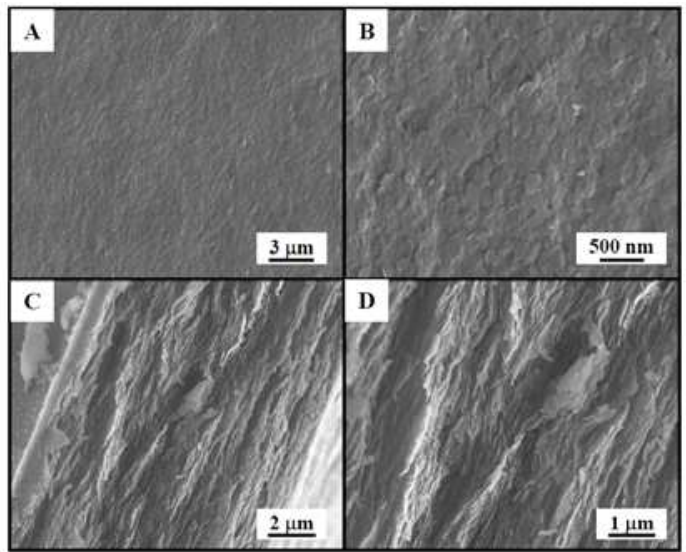

Fig. 11. SEM images of PEI-gibbsite nanocomposite. (A) Top-view image, (B) magnified topview image, $(C)$ cross-sectional image, and $(\mathrm{D})$ magnified cross-sectional image. Adapted from Lin, Huang et al. 2009.

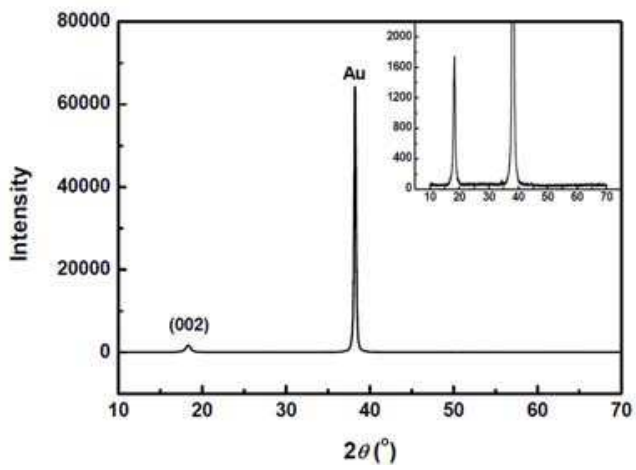

Fig. 12. XRD patterns of an electrodeposited PEI-gibbsite nanocomposite on Au electrode. Adapted from Lin, Huang et al. 2009.

TGA is carried out to determine the weight fraction of the organic phase in the nanocomposites shown in Fig. 13. An apparent thermal degradation process occurs at $\sim 250$ ${ }^{\circ} \mathrm{C}$ that corresponds to the degradation of the polymer matrix and the decomposition reaction of gibbsite. Based on the residual mass percentage $(63.7 \%)$ and assuming the ash contains only $\mathrm{Al}_{2} \mathrm{O}_{3}$, the weight fraction of PEI in the nanocomposite film is estimated to be $\sim 0.03$, which is close to the organic content of natural nacre consisting of less than $5 \mathrm{wt} \%$ of soft biological macromolecules. 


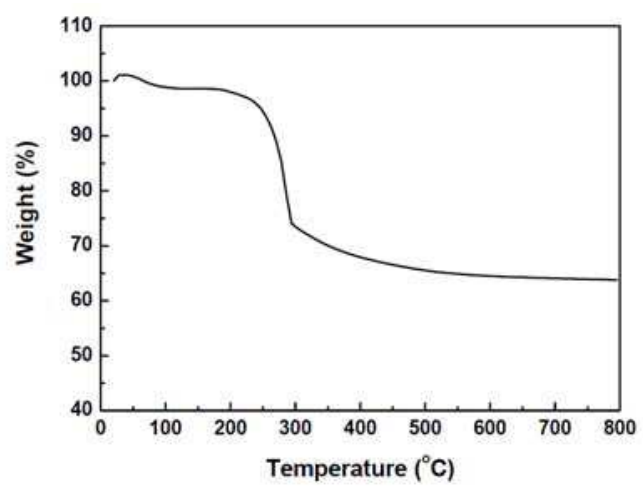

Fig. 13. Thermogravimetric analysis of an electrodeposited PEI-gibbsite nanocomposite. Adapted from Lin, Huang et al. 2009.

\subsection{Mechanical test}

The mechanical properties of the electrodeposited nanocomposites are evaluated using nanoindentation. This technique has been widely used in the characterization of mechanical behaviors of thin films, superhard coatings and nacres. In a nanoindentation test, a diamond Berkovich indenter is forced perpendicularly into the coating surface. The loaddisplacement profile is obtained during one cycle of loading and unloading, from which the hardness, $\mathrm{H}$, and the reduced modulus, $\mathrm{E}_{\mathrm{r}}$, are calculated using the Oliver-Pharr method(Oliver and Pharr 1992). In this method, the unloading curve is fitted to the powerlaw relation. The contact stiffness, $\mathrm{S}$, is then obtained by differentiating the power-law function at the maximum depth of penetration, $h_{\max }$. The contact depth, $h_{c}$, can be estimated from the load-displacement profile and then the contact area, A, is obtained by using empirically determined indenter shape function, $A=f(h)$, at $h_{c}$. Once the contact area is determined, the hardness, $H$, and reduced modulus, $E_{r}$, are obtained.

Fig. 14 shows the $E_{\mathrm{r}}$ as a function of contact depth obtained from the nanoindentation tests. The observed $E_{r}$ is in the range of 2.20 to $5.17 \mathrm{GPa}$. The decrease in $\mathrm{E}_{\mathrm{r}}$ with increasing contact

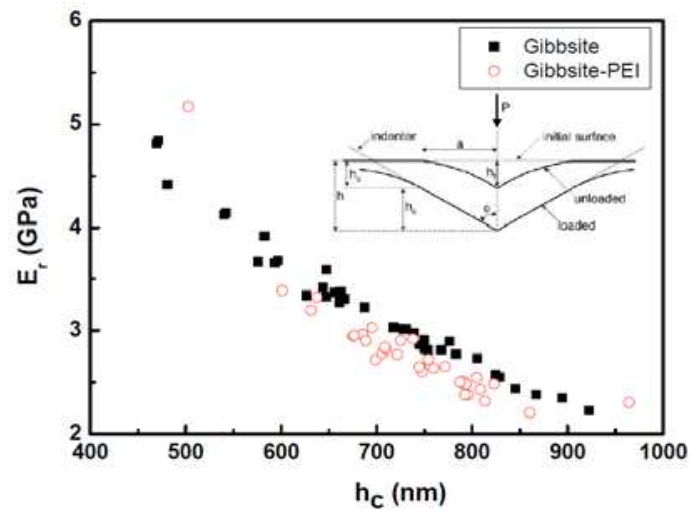

Fig. 14. Reduced modulus of pure gibbsite and PEI-gibbsite nanocomposite measured by nanoindentation. Adapted from Lin, Huang et al. 2009. 
depth may be related to the indentation size effects. The size effects are explained as a result of deformation, which is mainly from crack propagation for ceramics, and factors such as surface roughness, interaction between inorganic and organic phases, and other structural details of the coatings(Page, Oliver et al. 1992; Pharr 1998). The $E_{r}$ of PEI-gibbsite nanocomposite is $\sim 0.4 \mathrm{GPa}$ lower than that of pure gibbsite coating, showing the effect of the soft PEI layers in between the hard gibbsite nanoplatelets(Katti, Mohanty et al. 2006).

\section{Conclusion}

In conclusion, we have developed a simple and rapid electrodeposition technology for assembling gibbsite nanoplatelets into large-area, self-standing films. These nanosheets with high aspect ratio are preferentially aligned parallel to the electrode surface. The interstitials between the assembled nanoplatelets can be infiltrated with polymer to form optically transparent nanocomposites. The tensile strength and the stiffness of these biomimetic composites are significantly improved when compared to pure polymer films. The current electrodeposition technology is also promising for developing layered metal-ceramic and conducting polymer-ceramic nanocomposites that may exhibit improved mechanical and electrical properties but are not easily available by other bottom-up technologies (e.g., LBL assembly). We have also demonstrated that rapid production of nacre-like inorganic-organic nanocomposites can be achieved in a single step by electrophoretic co-deposition technology. The resulting self-standing polymer-gibbsite films are optically transparent and flexible. This technology is readily applicable to many other polyelectrolyte-nanoplatelet systems.

\section{References}

Aksay, I. A., M. Trau, et al. (1996). "Biomimetic pathways for assembling inorganic thin films." Science 273(5277): 892-898.

Almqvist, N., N. H. Thomson, et al. (1999). "Methods for fabricating and characterizing a new generation of biomimetic materials." Mater. Sci. Eng. C 7(1): 37-43.

Barthelat, F. (2007). "Biomimetics for next generation materials." Phil. Trans. R. Soc. A 365: 2907-2919.

Bonderer, L. J., A. R. Studart, et al. (2008). "Bioinspired design and assembly of platelet reinforced polymer films." Science 319(5866): 1069-1073.

Braun, P. V. and P. Wiltzius (1999). "Microporous materials - Electrochemically grown photonic crystals." Nature 402(6762): 603-604.

Brown, A. B. D., S. M. Clarke, et al. (1998). "Ordered phase of platelike particles in concentrated dispersions." Langmuir 14(11): 3129-3132.

Chen, R. F., C. A. Wang, et al. (2008). "An efficient biomimetic process for fabrication of artificial nacre with ordered-nano structure." Mater. Sci. Eng. C 28(2): 218-222.

Cullity, B. D. (1978). Elements of x-ray diffraction. Reading, MA, Addison-Wesley Publishing Company.

Dietrich, A. and A. Neubrand (2001). "Effects of particle size and molecular weight of polyethylenimine on properties of nanoparticulate silicon dispersions." J. Am. Ceram. Soc. 84(4): 806-812. 
Grandfield, K. and I. Zhitomirsky (2008). "Electrophoretic deposition of composite hydroxyapatite-silica-chitosan coatings." Mater. Character. 59(1): 61-67.

Holgado, M., F. Garcia-Santamaria, et al. (1999). "Electrophoretic deposition to control artificial opal growth." Langmuir 15(14): 4701-4704.

Jackson, A. P., J. F. V. Vincent, et al. (1988). "THE MECHANICAL DESIGN OF NACRE." Proc. R. Soc. Lond. B 234(1277): 415-\&.

Katti, K. S., B. Mohanty, et al. (2006). "Nanomechanical properties of nacre." J. Mater. Res. 21(5): 1237-1242.

Lin, T. H., W. H. Huang, et al. (2009). "Bioinspired Assembly of Colloidal Nanoplatelets by Electric Field." Chem. Mater. 21(10): 2039-2044.

Lin, T. H., W. H. Huang, et al. (2009). "Electrophoretic co-deposition of biomimetic nanoplatelet-polyelectrolyte composites." Electrochem. Commun. 11: 1635-1638.

Lin, T. H., W. H. Huang, et al. (2009). "Electrophoretic deposition of biomimetic nanocomposites." Electrochem. Commun. 11(1): 14-17.

Liu, T., B. Q. Chen, et al. (2008). "Ordered assemblies of clay nano-platelets." Bioinsp. Biomim. 3: 016005.

Macleod, H. A. (2001). Thin-Film Optical Filters. Bristol, Institute of Physics Publishing.

Mourad, M. C. D., J. Wijnhoven, et al. (2006). "Gelation versus liquid crystal phase transitions in suspensions of plate-like particles." Phil. Trans. R. Soc. A 364(1847): 2807-2816.

Munch, E., M. E. Launey, et al. (2008). "Tough, Bio-Inspired Hybrid Materials." Science 322(5907): 1516-1520.

Oliver, W. C. and G. M. Pharr (1992). "An improved technique for determining hardness and elastic-modulus using load and displacement sensing indentation experiments." J. Mater. Res. 7(6): 1564-1583.

Page, T. F., W. C. Oliver, et al. (1992). "The deformation-behavior of ceramic crystals subjected to very low load (nano)indentations." J. Mater. Res. 7(2): 450-473.

Pang, X. and I. Zhitomirsky (2008). "Electrodeposition of hydroxyapatite-silver-chitosan nanocomposite coatings." Surf. Coatings Technol. 202(16): 3815-3821.

Pharr, G. M. (1998). "Measurement of mechanical properties by ultra-low load indentation." Mater. Sci. Eng. A 253(1-2): 151-159.

Podsiadlo, P., A. K. Kaushik, et al. (2007). "Ultrastrong and stiff layered polymer nanocomposites." Science 318: 80-83.

Podsiadlo, P., M. Michel, et al. (2008). "Exponential growth of LBL films with incorporated inorganic sheets." Nano Lett. 8(6): 1762-1770.

Smith, B. L., T. E. Schaffer, et al. (1999). "Molecular mechanistic origin of the toughness of natural adhesives, fibres and composites." Nature 399(6738): 761-763.

Tang, Z. Y., N. A. Kotov, et al. (2003). "Nanostructured artificial nacre." Nat. Mater. 2(6): 413$\mathrm{U} 8$.

van der Beek, D. and H. N. W. Lekkerkerker (2004). "Liquid crystal phases of charged colloidal platelets." Langmuir 20(20): 8582-8586.

van der Beek, D., P. B. Radstake, et al. (2007). "Fast formation of opal-like columnar colloidal crystals." Langmuir 23: 11343-11346. 
van der Kooij, F. M., K. Kassapidou, et al. (2000). "Liquid crystal phase transitions in suspensions of polydisperse plate-like particles." Nature 406(6798): 868-871.

van der Kooij, F. M. and H. N. W. Lekkerkerker (1998). "Formation of nematic liquid crystals in suspensions of hard colloidal platelets." J. Phys. Chem. B 102(40): 7829-7832.

Velev, O. D. and K. H. Bhatt (2006). "On-chip micromanipulation and assembly of colloidal particles by electric fields." Soft Matter 2(9): 738-750.

Wierenga, A. M., T. A. J. Lenstra, et al. (1998). "Aqueous dispersions of colloidal gibbsite platelets: synthesis, characterisation and intrinsic viscosity measurements." Colloids Surf. A 134(3): 359-371.

Zhitomirsky, I. (2002). "Cathodic electrodeposition of ceramic and organoceramic materials. Fundamental aspects." Adv. Colloid Interface Sci. 97(1-3): 279-317. 


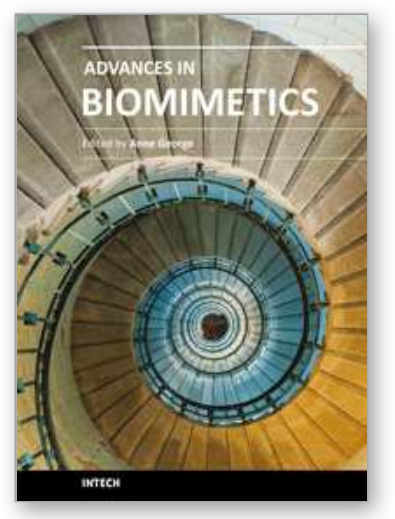

\author{
Advances in Biomimetics \\ Edited by Prof. Marko Cavrak
}

ISBN 978-953-307-191-6

Hard cover, 522 pages

Publisher InTech

Published online 26, April, 2011

Published in print edition April, 2011

The interaction between cells, tissues and biomaterial surfaces are the highlights of the book "Advances in Biomimetics". In this regard the effect of nanostructures and nanotopographies and their effect on the development of a new generation of biomaterials including advanced multifunctional scaffolds for tissue engineering are discussed. The 2 volumes contain articles that cover a wide spectrum of subject matter such as different aspects of the development of scaffolds and coatings with enhanced performance and bioactivity, including investigations of material surface-cell interactions.

\title{
How to reference
}

In order to correctly reference this scholarly work, feel free to copy and paste the following:

Tzung-Hua Lin, Wei-Han Huang, In-kook Jun and Peng Jiang (2011). Bioinspired Assembly of Inorganic Nanoplatelets for Reinforced Polymer Nanocomposites, Advances in Biomimetics, Prof. Marko Cavrak (Ed.), ISBN: 978-953-307-191-6, InTech, Available from: http://www.intechopen.com/books/advances-inbiomimetics/bioinspired-assembly-of-inorganic-nanoplatelets-for-reinforced-polymer-nanocomposites

\section{INTECH}

open science | open minds

\section{InTech Europe}

University Campus STeP Ri

Slavka Krautzeka 83/A

51000 Rijeka, Croatia

Phone: +385 (51) 770447

Fax: +385 (51) 686166

www.intechopen.com

\section{InTech China}

Unit 405, Office Block, Hotel Equatorial Shanghai

No.65, Yan An Road (West), Shanghai, 200040, China

中国上海市延安西路65号上海国际贵都大饭店办公楼405单元

Phone: +86-21-62489820

Fax: +86-21-62489821 
(C) 2011 The Author(s). Licensee IntechOpen. This chapter is distributed under the terms of the Creative Commons Attribution-NonCommercialShareAlike-3.0 License, which permits use, distribution and reproduction for non-commercial purposes, provided the original is properly cited and derivative works building on this content are distributed under the same license. 